\title{
Simulation of the Lock-Exchange Hydraulics Using the Discontinuous Galerkin Method
}

\author{
Nouh Izem \\ EMMS Faculty of science, Ibn \\ Zohr University Agadir, \\ Morocco
}

\author{
Mohammed Seaid \\ School of Engineering and \\ Computing Sciences, University \\ of Durham, South Road, \\ Durham DH1 3LE, UK
}

\author{
Mohamed Wakrim \\ EMMS Faculty of science, Ibn \\ Zohr University Agadir, \\ Morocco
}

\begin{abstract}
Numerical simulations of the lock-exchange hydraulics have been carried out using a discontinuous Galerkin finite element method. The basic water circulation in the lock-exchange hydraulics consists in an upper layer of cold, fresh surface water and an opposite deep current of warmer, salty outflowing water. The governing equations are the wellestablished two-layer shallow water system including bathymetric forces. The considered discontinuous Galerkin method is a stable, highly accurate and locally conservative finite element method whose approximate solutions are discontinuous across interelement boundaries; this property renders the method ideally suited for the hp-adaptivity. The proposed method can handle complex topography using unstructured grids and it satisfies the conservation property. Several numerical results are presented to demonstrate the high resolution of the proposed method and to confirm its capability to provide accurate and efficient simulations for the lock-exchange hydraulics.
\end{abstract}

\section{Keywords}

Discontinuous Galerkin method; Two-layer shallow water equations; Finite element; Lock-exchange hydraulics.

\section{INTRODUCTION}

During the last decades partial differential equations have been used as practical tools to model many environmental problems from real life. They have also been used to approximate and predict the dynamics of such problems. The goal of the present work is to provide a highly accurate and practical numerical model able to resolve and correctly capture the lock-exchange hydraulics. The water flow is governed by the depth-averaged Navier-Stokes equations involving several assumptions including (i) the domain is shallow enough to ignore the vertical effects, (ii) the pressure is hydrostatic, and (iii) viscous dissipation of the energy is ignored. These shallow water equations in depth-averaged form have been successfully applied to many engineering problems and their application fields include a wide spectrum of phenomena other than water waves. For instance, the shallow water equations have applications for tidal flows in an estuary or coastal regions, rivers, reservoirs and open channel flows. Such practical flow problems are not trivial to simulate since the geometry can be complex and the topography irregular. On the other hand, single-layer shallow water equations have the drawback of missing some physical dynamics in the vertical motion. Therefore, during the past years, multi-layer shallow water models have been attracted more attention and have became a very useful tools to solve hydrodynamical flows such as rivers, estuaries, bays and other nearshore regions where water flows interact with the bed geometry and wind shear stresses, see for instance $[12,5,22$, $1,10,23]$. The layers can be formed in the shallow water model based on the vertical variation of water density which in general depends on the water temperature and water salinity. The main advantage of these models is the fact that the two-layer shallow water model avoids the expensive threedimensional Navier-Stokes equations and obtains stratified horizontal flow velocities as vertical velocities are relatively small and the flow is still within the shallow water regime. Accurate modeling of the lock-exchange hydraulics requires numerical methods capable of capturing highly advective flows and multi-scale features of the solution. The emphasis of the present work is on the application of the so-called nodal Discontinuous Galerkin (DG) methods to the lock-exchange hydraulics. The nodal DG method first introduced by Hesthaven and Warburton [16] for electro-dynamic simulations utilizes a nodal Lagrange interpolation basis as the approximating basis functions, which provides a simple and generic means to treat a (nonlinear) flux term appearing in the hyperbolic conservation laws. In recent years, a multitude of DG formulations has found rapid applications in many fields, for a review we refer to $[6,8]$ and further references are therein. The DG method has been successfully applied to the standard single-layer shallow water equations, see for example $[2,11,21,14]$. However, the presence of nonconservative product terms in their two-layer counterpart poses serious numerical problems and at present there is no literature available how to genuinely solve the two-layer shallow water equations in a DG context, which motivated the research discussed in this article. Results presented in this paper show high resolution of the proposed DG method and confirm its capability to provide robust and accurate simulations for two-layer shallow water flows including complex topography.

The structure of this paper is as follows. In section 2, we present the mathematical equations for the two-layer shallow water equations used to model the lock-exchange hydraulics. The formulation of the DG method is detailed in section 3 . This section includes the finite element discretization, the formulation of the weak form, numerical fluxes, and the time integration scheme. Section 4 is devoted to numerical results and applications. Finally, section 5 contains the conclusions.

\section{Governing Equations for Two-layer Shallow Water Problems}

The two-layer shallow water equations are derived from the depth-averaged incompressible Navier-Stokes or Euler equations, compare [30] among others. For two-dimensional flow problems, these equations are given by 


$$
\begin{gathered}
\frac{\partial h_{1}}{\partial t}+\frac{\partial\left(h_{1} u_{1}\right)}{\partial x}+\frac{\partial\left(h_{1} v_{1}\right)}{\partial y}=0, \\
\frac{\partial\left(h_{1} u_{1}\right)}{\partial t}+\frac{\partial}{\partial x}\left(h_{1} u_{1}^{2}+\frac{1}{2} g h_{1}^{2}\right)+\frac{\partial}{\partial y}\left(h_{1} u_{1} v_{1}\right)= \\
-g h_{1} \frac{\partial}{\partial x}\left(h_{2}+B\right), \\
\frac{\partial\left(h_{1} v_{1}\right)}{\partial t}+\frac{\partial}{\partial x}\left(h_{1} u_{1} v_{1}\right)+\frac{\partial}{\partial y}\left(h_{1} v_{1}^{2}+\frac{1}{2} g h_{1}^{2}\right)= \\
-g h_{1} \frac{\partial}{\partial y}\left(h_{2}+B\right), \\
\frac{\partial h_{2}}{\partial t}+\frac{\partial\left(h_{2} u_{2}\right)}{\partial x}+\frac{\partial\left(h_{2} v_{2}\right)}{\partial y}=0 \\
+\frac{\partial}{\partial x}\left(h_{2} u_{2}{ }^{2}+\frac{1}{2} g h_{2}{ }^{2}\right)+\frac{\partial}{\partial y}\left(h_{2} u_{2} v_{2}\right)= \\
-g h_{2} \frac{\partial}{\partial x}\left(\frac{\rho_{1}}{\rho_{2}} h_{1}+B\right), \\
\frac{\partial\left(h_{2} v_{2}\right)}{\partial t}+\frac{\partial}{\partial x}\left(h_{2} u_{2} v_{2}\right)+\frac{\partial}{\partial y}\left(h_{2} v_{2}{ }^{2}+\frac{1}{2} g h_{2}{ }^{2}\right)= \\
-g h_{2} \frac{\partial}{\partial y}\left(\frac{\rho_{1}}{\rho_{2}} h_{1}+B\right),
\end{gathered}
$$

where the subscripts 1 and 2 represent respectively, the upper and lower layer in the hydraulic system. In the equations (1)(2), $\rho_{j}$ is the water density of the $j$ th layer, $h_{j}(x, y, t)$ is the water height of the $j$ th layer, $u_{j}(x, y, t)$ and $v_{j}(x, y, t)$ are respectively, the depth-averaged water velocities in $x$ - and $y$ direction for the $j$ th layer, with $j=1,2, B(x, y)$ is the bottom topography and $g$ the gravitational acceleration. For simplicity in the presentation we can also reformulate the two-layer shallow water equations (1)-(2) in a matrix form as

$\frac{\partial \mathrm{W}}{\partial t}+\frac{\partial F(\mathrm{~W})}{\partial x}+\frac{\partial G(\mathrm{~W})}{\partial y}+A(\mathrm{~W}) \frac{\partial \mathrm{W}}{\partial x}+B(\mathrm{~W}) \frac{\partial \mathrm{W}}{\partial y}=S(\mathrm{~W})$,

where

$$
\begin{gathered}
\mathrm{W}=\left(h_{1}, q_{1, x}, q_{1, y}, h_{2}, q_{2, x}, q_{2, y}\right)^{T}, \\
S=\left(0,-\mathrm{gh}_{1} \frac{\partial \mathrm{B}}{\partial \mathrm{x}},-\mathrm{gh}_{1} \frac{\partial \mathrm{B}}{\partial \mathrm{y}}, 0,-\mathrm{gh}_{2} \frac{\partial \mathrm{B}}{\partial \mathrm{x}},-\mathrm{gh}_{2} \frac{\partial \mathrm{B}}{\partial \mathrm{y}}\right)^{T}, \\
A(W)=\left(\begin{array}{cccccc}
0 & 0 & 0 & 0 & 0 & 0 \\
g & 0 & 0 & g h_{1} & 0 & 0 \\
0 & 0 & 0 & 0 & 0 & 0 \\
0 & 0 & 0 & 0 & 0 & 0 \\
r g h_{2} & 0 & 0 & 0 & 0 & 0 \\
0 & 0 & 0 & 0 & 0 & 0
\end{array}\right), \quad B(W)=\left(\begin{array}{cccccc}
0 & 0 & 0 & 0 & 0 & 0 \\
g & 0 & 0 & 0 & 0 & 0 \\
0 & 0 & 0 & g h_{1} & 0 & 0 \\
0 & 0 & 0 & 0 & 0 & 0 \\
0 & 0 & 0 & 0 & 0 & 0 \\
r g h_{2} & 0 & 0 & 0 & 0 & 0
\end{array}\right),
\end{gathered}
$$

where $r=\frac{\rho_{1}}{\rho_{2}}$ is the density ratio, $q_{j, x}=h_{j} u_{j}$, and $q_{j, y}=$ $h_{j} v_{j}$, with $\mathrm{j}=1,2$, are the water discharges. The equations (3) have to be solved for a time interval $[0, T]$ in a bounded spatial domain $\Omega \subset \mathbb{R}^{2}$ with a boundary $\Gamma$, equipped with given boundary and initial conditions. In practice, boundary and initial conditions are problem dependent and their formulation is postponed to section 4 where numerical examples are discussed. It is well known that the calculation of the eigenvalues associated with the two-layer system (3) is not trivial. Indeed, there are six distinct eigenvalues in each of the $\mathrm{x}$ - and $\mathrm{y}$-directions respectively such that the corresponding eigenvectors are linearly independent. Two of the eigenvalues are given by

$$
\lambda_{1}=U_{1}, \quad \lambda_{2}=U_{2},
$$

where $U_{\mathrm{j}}=u_{j} n_{x}+v_{j} n_{y}$, with $j=1,2$, is the velocity across the element face in the respective layer. The other four eigenvalues $\lambda_{k}(k=3, \ldots, 6)$ are the zeros of the characteristic polynomial

$$
\begin{gathered}
P(\lambda)=\left(\lambda^{2}-2 U_{1} \lambda+U_{1}{ }^{2}-g h_{1}\right)\left(\lambda^{2}-2 U_{2} \lambda+U_{2}{ }^{2}\right. \\
\left.-g h_{2}\right)-g^{2} r h_{1} h_{2} .
\end{gathered}
$$

Here, $\mathrm{n}=\left(n_{x}, n_{y}\right)^{\mathrm{T}}$ is the unit outward normal vector. For hydraulic applications with $r \approx 1$ and $U_{1} \approx U_{2}$, a first-order approximation of the eigenvalues can be obtained by expanding (4) in terms of $1-r$ and $U_{2}-U_{1}$ as

and

$$
\begin{aligned}
& \lambda_{3} \approx V_{m}-\sqrt{g\left(h_{1}+h_{2}\right)}, \\
& \lambda_{4} \approx V_{m}+\sqrt{g\left(h_{1}+h_{2}\right)},
\end{aligned}
$$

$$
\begin{aligned}
& \lambda_{5} \approx V_{c}-\sqrt{g^{\prime} \frac{h_{1} h_{2}}{h_{1}+h_{2}}\left(1-\frac{\left(U_{2}-U_{1}\right)^{2}}{g^{\prime}\left(h_{1}+h_{2}\right)}\right)}, \\
& \lambda_{6} \approx V_{c}+\sqrt{g^{\prime} \frac{h_{1} h_{2}}{h_{1}+h_{2}}\left(1-\frac{\left(U_{2}-U_{1}\right)^{2}}{g^{\prime}\left(h_{1}+h_{2}\right)}\right)},
\end{aligned}
$$

where $g^{\prime}=(1-r) g$ is the reduced gravity, $V_{m}$ is the mean velocity and $V_{c}$ is the convective velocity defined by

$$
V_{m}=\frac{h_{1} U_{1}+h_{1} U_{1}}{h_{1}+h_{2}} \quad V_{c}=\frac{h_{1} U_{2}+h_{2} U_{1}}{h_{1}+h_{2}}
$$

It is evident that, depending on the values of the ratio $r$, the eigenvalues (7) may become complex. In this case, the system is not hyperbolic and yields to the so-called Kelvin-Helmholtz instability at the interface separating the two layers. A necessary condition for the system (3) to be hyperbolic is

$$
\frac{\left(U_{2}-U_{1}\right)^{2}}{g^{\prime}\left(h_{1}+h_{2}\right)}<1
$$

It should be stressed that the DG method does not require explicit calculation of the eigenvalues of the system (3). A simplified approximation of these eigenvalues could be used in the reconstruction of numerical fluxes as well as in the selection of time steps for the time integration procedure.

\section{DISCONTINUOUS GALERKIN METHOD}

The two-layer shallow water system (3) is numerically solved using a splitting operator where the spatial discretization and the time integration are performed separately using the method of lines. In this section, we first discuss a discontinuous Galerkin finite element method for the spatial discretization. Then a third-order Runge-Kutta time integration scheme is formulated for the semi-discrete system. It should be stressed that the main idea of the DG method is to allow the finite element solution to be discontinuous over the boundaries of the local element and it only couples adjacent elements using suitable numerical fluxes, see for instance [6] and further references are therein. The selection of numerical fluxes in this method can be borrowed from the finite volume techniques, which is theoretically well-established. Note that the DG method combined with nodes located at the boundaries offers the opportunity to determine the numerical fluxes directly. 


\subsection{The special discretization}

The computational domain $\Omega_{h}$ is divided into $N_{e}$ nonoverlapping elements, such that $\Omega_{h}=\mathrm{U}_{k=1}^{N_{e}} \mathcal{K}_{k}$, where $N_{e}$ the number of elements of and $h$ is is a space discretization parameter. We introduce the following broken Sobolev space

$$
\mathbb{V}_{k}^{N}=\left\{v: \quad v_{k} \in \mathbb{P}^{N}\left(\mathcal{K}_{k}\right), \forall \mathcal{K}_{k} \in \Omega_{h}\right\},
$$

where denotes the set of polynomials of degree up to $N$ defined on the element $\mathcal{K}_{k}$. To perform differentiation and integration operations, we introduce the non-singular mapping, $\Psi$, connecting the general straight-sided triangle $\mathcal{K}_{k}$ with the standard straight-angle. The mapping $\Psi$ is defined as

$$
\mathfrak{I}=\{\mathbf{r}=(r, s) \in[-1,1]: \quad r+s \leq 0\},
$$

Before applying the discontinuous Galerkin procedure we reformulate the equations (3) in the compact form

$$
\frac{\partial \mathrm{W}}{\partial t}+\nabla \cdot \mathcal{F}(\mathrm{W})=\mathcal{Q}(\mathrm{W})
$$

where the flux function $\mathcal{F}(\mathrm{W})=(\mathrm{F}(\mathrm{W}), \mathrm{G}(\mathrm{W}))^{\mathrm{T}}$ and the source term

$$
\mathcal{Q}(\mathrm{W})=S(\mathrm{~W})-A(\mathrm{~W}) \frac{\partial \mathrm{W}}{\partial x}-B(W) \frac{\partial \mathrm{W}}{\partial y}
$$

and we start by assuming that one can represent the global solution of (10) as a direct sum of local piecewise polynomial solution as

$$
\mathrm{W}(\mathbf{x}, t) \simeq \mathrm{W}_{h}(\mathbf{x})=\bigoplus_{k=1}^{N_{e}} \mathrm{~W}_{h}^{k}(\mathbf{x}),
$$

where the solution on the triangular element $\mathcal{K}_{k}$ is locally approximated by

$W^{k}(\mathbf{x}) \simeq W_{h}^{k}(\mathbf{x})=\sum_{n=1}^{N_{p}} \widetilde{W}_{n}^{k}(t) \psi_{n}(\mathbf{x})=\sum_{i=1}^{N_{p}} W_{h}^{k}\left(t, \mathbf{x}_{i}\right) \mathcal{L}_{i}(\mathbf{x})$

where we have $N_{p}=(N+1)(N+2) / 2$ degrees of freedom inside each element in terms of the unknown modal coefficients $\widetilde{\mathrm{W}}_{\mathrm{n}}^{\mathrm{k}}$ or nodal coefficients $\mathrm{W}_{\mathrm{h}}^{\mathrm{k}}\left(\mathrm{t}, \mathrm{x}_{\mathrm{i}}\right)$ for $n, i=$ $1, \ldots N_{p}, \quad \psi_{n}$ is the easily constructed Proriol-KoornwinderDubiner orthonomal (PKD) basis functions [27, 20, 9] and $\mathcal{L}_{i}(\mathbf{x})$ are the two-dimensional Lagrange polynomials defined on the set of nodes $x_{i}$ used in combination with the chosen orthogonal basis $\psi_{n}$. The details on the construction of the Lagrange polynomial basis functions can be found in [13] where cardinal functions based on the PKD polynomials are used.

$$
\psi_{m}(r, s)=\sqrt{2} P_{\mathrm{i}}^{(0,0)}(a) P_{\mathrm{i}}^{(2 \mathrm{i}+1,0)}(b)(1-b)^{i},
$$

where $P_{\mathrm{n}}^{(\alpha, \beta)} \mathrm{n}$ is the $n$ th-order Jacobi polynomial and $a=2 \frac{1+r}{1-s}, \quad b=s, \quad m=i+(N+1) j+1-\frac{j}{2}(j-1)$. For the interpolation points $\mathbf{x}_{\mathrm{i}}=\Psi\left(\mathrm{r}_{\mathrm{i}}, \mathrm{s}_{\mathrm{i}}\right)$ we choose the nodal set derived from the electrostatics principle [15] for $\mathrm{N}<$ 11 and the Fekete points [25] for $11=N=15$. Note that this grid distribution becomes the Legendre-Gauss-Lobatto distribution along the edges of the triangle. We define the vectors of nodal and modal values on $\mathcal{K}_{k}$ as

$$
\widetilde{\mathrm{W}}_{n}^{\mathrm{k}}=\left(\widetilde{\mathrm{W}}_{1}^{\mathrm{k}}, \ldots, \widetilde{\mathrm{W}}_{N_{p}}^{\mathrm{k}}\right)^{\mathrm{T}}, \quad \mathrm{W}_{\mathrm{h}}^{\mathrm{k}}=\left(\mathrm{W}_{\mathrm{h}}^{\mathrm{k}}\left(r_{1}^{k}, s_{1}^{k}\right), \ldots, \mathrm{W}_{\mathrm{h}}^{\mathrm{k}}\left(r_{N_{p}}^{k}, s_{N_{p}}^{k}\right)\right)^{\mathrm{T}},
$$

and the vectors of local Lagrange polynomials and basis functions on $\mathcal{K}_{k}$ as

$$
\mathcal{L}=\left(\mathcal{L}_{1}, \ldots, \mathcal{L}_{N_{p}}\right)^{\mathrm{T}}, \boldsymbol{\psi}=\left(\psi_{1}, \ldots, \psi_{N_{p}}\right)^{\mathrm{T}} .
$$

This leaves us with the following relationship between the modal and nodal coefficients and basis functions

$$
\mathrm{W}_{\mathrm{h}}^{\mathrm{k}}=\mathrm{V} \widetilde{\mathrm{W}}_{n}^{\mathrm{k}}, \quad \boldsymbol{\psi}(\overline{\boldsymbol{r}}, \overline{\boldsymbol{s}})=V^{t} \boldsymbol{\mathcal { L }}(\overline{\boldsymbol{r}}, \overline{\boldsymbol{s}}),
$$

where we have defined the vectors $\overline{\boldsymbol{r}}=\left(r_{1}, \ldots, r_{N_{p}}\right)^{T}, \overline{\boldsymbol{s}}=$ $\left(s_{1}, \ldots, s_{N_{p}}\right)^{T}$, and $\mathrm{V}$ is the well-known Vandermonde matrix with entries $\mathrm{V}_{i, j}=\psi_{j}\left(r_{i}, s_{i}\right)$.

Following the DG-FEM procedure, for each element $\mathcal{K}_{k}$ in the domain we multiply the two-layer shallow water equations (10) with a test function $\mathcal{L}_{i}^{k}(\mathbf{x})$. Two integration by parts are carried out for the divergence term. In the intermediate step of these partial integrations, the analytic flux function $n_{x}^{k} F_{h}^{k}+n_{y}^{k} G_{h}^{k}$ is interchanged with a continuous numerical flux function $\mathcal{F}^{k^{*}}=\left(n_{x}^{k} F_{h}^{k}+n_{y}^{k} G_{h}^{k}\right)^{*}$ to be chosen, which allow us to connect adjacent elements. By this approach, the starting point for the strong DG formulation of (10) for the $k$ th element, $k=1, \ldots, N_{\mathrm{e}}$ and $m=1, \ldots, N_{\mathrm{p}}$, becomes

$$
\begin{gathered}
\int_{\mathcal{K}_{k}}\left(\frac{\partial \mathrm{W}_{\mathrm{h}}^{\mathrm{k}}}{\partial t}+\nabla \cdot \mathcal{F}_{h}^{k}\right) \mathcal{L}_{m}^{k} d \mathbf{x} \quad=\oint_{\partial \mathcal{K}_{k}}\left(\mathrm{n}^{k} \cdot \mathcal{F}_{h}^{k}-\mathrm{n}^{k} \cdot \mathcal{F}^{*}\right) \mathcal{L}_{m}^{k} d \mathbf{x} \\
+\int_{\mathcal{K}_{k}} \mathcal{Q}_{\mathrm{h}}^{\mathrm{k}} \mathcal{L}_{m}^{k} d \mathbf{x}
\end{gathered}
$$

In the present work, we consider the local monotone LaxFriedrichs flux defined by [24]

$$
\mathcal{F}^{*}\left(\mathrm{~W}_{\mathrm{h}}^{-}, \mathrm{W}_{\mathrm{h}}^{+}\right)=\frac{\left(\mathcal{F}\left(\mathrm{W}_{\mathrm{h}}^{-}\right)+\mathcal{F}\left(\mathrm{W}_{\mathrm{h}}^{+}\right)\right)}{2}+\frac{C}{2}\left(\mathrm{~W}_{\mathrm{h}}^{-}-\mathrm{W}_{\mathrm{h}}^{+}\right),
$$

where $\mathrm{W}_{\mathrm{h}}^{-}$refers to the local solution, $\mathrm{W}_{\mathrm{h}}^{+}$refers to the neighboring solution, and $C$ is the local maximum of the directional flux Jacobian defined as

$$
C=\max _{W \in\left[\mathrm{w}_{\mathrm{h}}^{-}, \mathrm{w}_{\mathrm{h}}^{+}\right]}\left|\mathrm{n}^{k} \cdot \frac{\partial F}{\partial W}\right| .
$$

The local monotone Lax-Friedrichs numerical flux is a particularly convenient choice of numerical flux because it can be easily applied to any non-linear hyperbolic system, it is simple to compute, and yields good results, although there are many other numerical fluxes which could also be used [18].

Using the polynomial approximation (11) for $\mathrm{W}_{\mathrm{h}}^{\mathrm{k}}, F_{h}^{k}, G_{h}^{k}$ and $Q_{\mathrm{h}}^{\mathrm{k}}$ the strong DG formulation (14) becomes,

$$
\begin{aligned}
\sum_{i=1}^{N_{p}} \int_{\mathcal{K}_{k}} \frac{\partial W_{h}^{k}}{\partial t}\left(\mathbf{x}_{i}^{k}\right) \mathcal{L}_{j}^{k}(\mathbf{x}) \mathcal{L}_{i}^{k}(\mathbf{x}) d \mathbf{x}= \\
\quad-\sum_{i=1}^{N_{p}} \int_{\mathcal{K}_{k}}\left(F_{h}^{k}\left(\mathbf{x}_{i}^{k}\right) \frac{\partial \mathcal{L}_{j}^{k}}{\partial x}(\mathbf{x})+G_{h}^{k}\left(\mathbf{x}_{i}^{k}\right) \frac{\partial \mathcal{L}_{j}^{k}}{\partial y}(\mathbf{x})\right) \mathcal{L}_{i}^{k}(\mathbf{x}) d \mathbf{x} \\
+\oint_{\partial \mathcal{K}_{k}}\left(\left(n_{x}^{k} F_{h}^{k}+n_{y}^{k} G_{h}^{k}\right)-\mathrm{n}^{k} \cdot\left(n_{x}^{k} F_{h}^{k}+n_{y}^{k} G_{h}^{k}\right)^{*}\right) \mathcal{L}_{i}^{k}(\mathbf{x}) d \mathbf{x} \\
+\sum_{\mathrm{i}=\mathbf{1}}^{N_{p}} \int_{\mathcal{K}_{k}} Q_{\mathrm{h}}^{\mathrm{k}} \mathcal{L}_{j}^{k}(\mathbf{x}) \mathcal{L}_{i}^{k}(\mathbf{x}) d \mathbf{x} .
\end{aligned}
$$

Next, note that by defining the following discrete elemental operators

$$
M_{i, j}^{k}=\int_{\mathcal{K}_{k}} \mathcal{L}_{j}^{k} \mathcal{L}_{i}^{k} d \mathbf{x}, S_{i, j}^{k}=\int_{\mathcal{K}_{k}} \mathcal{L}_{j}^{k} \nabla \mathcal{L}_{i}^{k} d \mathrm{x}, M_{i, j}^{s}=\int_{\partial \mathcal{K}_{k}} \mathcal{L}_{j}^{k} \mathcal{L}_{i}^{k} d \mathbf{x},
$$

We can now write the semi-discrete system above for $\mathrm{k}=1, \ldots, \mathrm{N}_{\mathrm{e}}$ in the following matrix form:

$M_{i, j}^{k} \frac{\partial \mathrm{W}_{\mathrm{h}}^{\mathrm{k}}}{\partial t}+\left(S_{i, j}^{k}\right)^{T}\left(\mathcal{F}_{h}^{k}\right)_{j}-M_{i, j}^{k} Q_{h}^{k}=\left(M_{i, j}^{s}\right)^{T}\left(\mathcal{F}_{h}^{k}-\mathcal{F}^{*}\right)_{j}^{k}$

Let us now consider the evaluation of the mass matrix $\mathrm{M}$ and the stiffness matrix $\mathrm{S}$ for the standard element $\mathfrak{T}$ : 


$$
\begin{aligned}
M_{i, j}^{k} & =J^{k} \int_{\mathfrak{I}} \mathcal{L}_{i} \mathcal{L}_{j} d \mathbf{r} \equiv J^{k}(M)_{i, j}, \\
S_{i, j}^{k} & =J^{k} \int_{\mathfrak{T}} \mathcal{L}_{i} \nabla_{\mathrm{r}} \mathcal{L}_{j} \frac{\partial \mathbf{r}}{\partial \mathbf{x}} d \mathbf{r} \equiv J^{k}(M)_{i, j}, \\
& =J^{k}\left[\left(\frac{\partial r}{\partial x} \mathbf{i}+\frac{\partial r}{\partial y} \mathbf{j}\right) \int_{\mathfrak{T}} \mathcal{L}_{i} \frac{\partial \mathcal{L}_{j}}{\partial r} d \mathbf{r}+\left(\frac{\partial s}{\partial x} \mathbf{i}+\frac{\partial s}{\partial y} \mathbf{j}\right) \int_{\mathfrak{T}} \mathcal{L}_{i} \frac{\partial \mathcal{L}_{j}}{\partial s} d \mathbf{r}\right], \\
& \equiv J^{k} M_{i j}^{k}\left[\left(r_{x} D_{i j}^{r}+s_{x} D_{i j}^{s}\right) \mathbf{i}+\left(r_{y} D_{i j}^{r}+s_{y} D_{i j}^{s}\right) \mathbf{j}\right],
\end{aligned}
$$

where $\mathrm{J}^{\mathrm{k}}$ is the transformation Jacobian from the physical element to the reference one. The relationships (13) allow us to determine the stiffness matrix components from the following relations

$$
\begin{gathered}
D^{r}=\left.\frac{\partial \mathcal{L}(\mathrm{r}, \mathrm{s})}{\partial r}\right|_{r}=\left.\left(V^{t}\right)^{-1} \frac{\partial \psi(\mathrm{r}, \mathrm{s})}{\partial r}\right|_{r} \\
D^{s}=\left.\frac{\partial \mathcal{L}(\mathrm{r}, \mathrm{s})}{\partial s}\right|_{s}=\left.\left(V^{t}\right)^{-1} \frac{\partial \psi(\mathrm{r}, \mathrm{s})}{\partial s}\right|_{s}
\end{gathered}
$$

To evaluate the integrals over the faces $\Gamma_{i}^{k}(\mathrm{i}=1,2,3$ and $\mathrm{k}=$ $\left.1,2, \ldots, N_{\mathrm{e}}\right)$ of the triangular elements, we use the $1 \mathrm{D}$ interpolation $\mathcal{L}^{k, 1 \mathrm{D}}$ as

$$
\begin{aligned}
M_{i j}^{S} & =\sum_{n=1}^{3} \oint_{\Gamma_{n}^{k}} \mathcal{L}_{i}^{k, 1 \mathrm{D}} \mathcal{L}_{j}^{k, 1 \mathrm{D}} \mathrm{n}^{k} d \mathbf{x}=\sum_{n=1}^{3} J_{1 \mathrm{D}}^{k_{i}} \oint_{[-1,1]} \mathcal{L}_{i}^{k, 1 \mathrm{D}} \mathcal{L}_{j}^{k, 1 \mathrm{D}} \mathrm{n} d \mathbf{r} \\
& \equiv \sum_{n=1}^{3} J_{1 \mathrm{D}}^{k_{i}} M_{1 D}\left(n_{x} \boldsymbol{i}+n_{y} \boldsymbol{j}\right),
\end{aligned}
$$

with $J_{1 \mathrm{D}}$ is the transformation Jacobian along the face, the ratio between the length of the face in $\mathcal{K}_{k}$ and in $\mathfrak{T}$, respectively.

Finally, we obtain the following local semi-discrete equations on each triangle of the mesh

$$
\begin{aligned}
\frac{\partial \mathrm{W}_{\mathrm{h}}^{\mathrm{k}}}{\partial t}+\left(r_{x} D^{r}+s_{x} D^{s}\right) F_{h}^{k}+\left(r_{y} D^{r}+s_{y} D^{s}\right) G_{h}^{k}+ \\
\sum_{i=1}^{3} \frac{J_{1 \mathrm{D}}^{k_{i}}}{J^{k}}{ }^{-1} M_{1 \mathrm{D}}^{k_{i}}\left(\mathcal{F}^{k_{i}^{*}}-\mathcal{F}_{h}^{k_{i}}\right)=Q_{h}^{k}
\end{aligned}
$$

It is worth to mention that boundary conditions have to be incorporated in the semi-discrete system (19). In the DG framework it is usual to impose boundary conditions in a weak form in both, inflow and outflow, boundaries. It is recognized for most works published in the literature, that the weak imposition of Dirichlet-type conditions is superior to the strong imposition on outflow boundaries; see for instance [3]. This is due to the appearance of spurious oscillations in boundary layers when Dirichlet boundary conditions are imposed strongly. However, the weak enforcement of inflow Dirichlet boundary conditions offers no advantages over the strong imposition, compare for example [17] and further discussions are therein.

\subsection{Treatment of source term}

To approximate the source term, we assume that,

$$
B^{k}(\mathbf{x}) \simeq B_{h}^{k}(\mathbf{x})=\sum_{i=1}^{N_{p}} B_{h}^{k}\left(x_{i}, y_{i}\right) \mathcal{L}_{i}^{k}(\mathbf{x}),
$$

In the strong formulation, we recall the vector of nodal values

$$
Q_{h}^{k}=\left(Q_{h}^{k}\left(x_{1}^{k}, y_{1}^{k}\right), \ldots, Q_{h}^{k}\left(x_{N_{p}}^{k}, y_{N_{p}}^{k}\right)\right)^{\mathrm{T}},
$$

By using the differentiation matrices (17)-(18) and the chain rule we can form the discrete gradient operator as

$$
\frac{\partial}{\partial x}=r_{x}^{k} D^{r}+s_{x}^{k} D^{s}, \quad \frac{\partial}{\partial y}=r_{y}^{k} D^{r}+s_{y}^{k} D^{s} .
$$

which transforms point values, $B_{h}^{k}\left(x_{i}, y_{i}\right)$, to $x$-derivatives and $y$-derivatives respectively at these same points e.g.,

$$
\frac{\partial B_{h}^{k}}{\partial x}=\left[r_{x} D^{r}+s_{x} D^{s}\right] B_{h}^{k}, \quad \frac{\partial B_{h}^{k}}{\partial y}=\left[r_{y} D^{r}+s_{y} D^{s}\right] B_{h}^{k} .
$$

For brevity in presentation, we refer the reader to [19] where details about this kind of differentiation matrices are discussed in depth. Thus, the calculation of source term components will be obtained as following

$$
Q_{h}^{k}=\left(\begin{array}{c}
0 \\
-\operatorname{gh}_{1, h}^{k}\left[r_{x} D^{r}+s_{x} D^{s}\right]\left(B_{h}^{k}+\mathrm{h}_{2, h}^{k}\right) \\
-\operatorname{gh}_{1, h}^{k}\left[r_{y} D^{r}+s_{y} D^{s}\right]\left(B_{h}^{k}+\mathrm{h}_{2, h}^{k}\right) \\
0 \\
-\operatorname{gh}_{2, h}^{k}\left[r_{x} D^{r}+s_{x} D^{s}\right]\left(B_{h}^{k}+\frac{\rho_{1}}{\rho_{2}} \mathrm{~h}_{1, h}^{k}\right) \\
-\operatorname{gh}_{2, h}^{k}\left[r_{y} D^{r}+s_{y} D^{s}\right]\left(B_{h}^{k}+\frac{\rho_{1}}{\rho_{2}} \mathrm{~h}_{1, h}^{k}\right)
\end{array}\right)
$$

where

$$
\begin{aligned}
& \mathrm{h}_{i, h}^{k}=\left(h_{i, h}^{k}\left(x_{1}^{k}, y_{1}^{k}\right), \ldots, h_{i, h}^{k}\left(x_{N_{p}}^{k}, y_{N_{p}}^{k}\right)\right)^{\mathrm{T}}, i=1,2 \\
& B_{h}^{k}=\left(B_{h}^{k}\left(x_{1}^{k}, y_{1}^{k}\right), \ldots, B_{h}^{k}\left(x_{N_{p}}^{k}, y_{N_{p}}^{k}\right)\right)^{\mathrm{T}} .
\end{aligned}
$$

\subsection{The time integration}

The solution procedure for two-layer shallow water equations (3) is complete when a time integration of semi-discrete equations (19) is selected. In the current study, the time stepping scheme utilized is an explicit strong stability preserving (SSP) third-order Runge-Kutta scheme. The SSP Runge-Kutta scheme is designed so that if the forward Euler method is stable under a given semi-norm and CourantFriedrichs-Lewy (CFL) condition, then the higher-order scheme remains stable under the same semi-norm, but perhaps a different CFL condition, see [28] among others. This method also possesses the desirable total variation diminishing (TVD) property. By assembling together all the elemental contributions, the system (19) can be written as

$$
\begin{array}{r}
\frac{\partial \mathrm{W}_{\mathrm{h}}^{\mathrm{k}}}{\partial t}=-\left(r_{x} D^{r}+s_{x} D^{s}\right) F_{h}^{k}-\left(r_{y} D^{r}+s_{y} D^{s}\right) G_{h}^{k}- \\
\sum_{i=1}^{3} \frac{J_{1 \mathrm{D}}^{k_{i}}}{J^{k}}{ }^{-1} M_{1 \mathrm{D}}^{k_{i}}\left(\mathcal{F}^{k_{i}^{*}}-\mathcal{F}_{h}^{k_{i}}\right)+Q_{h}^{k}
\end{array}
$$

Let us rewrite the equations (24) in a compact ODE form as

$$
\begin{aligned}
\frac{d W}{d t} & =H(W), \quad t \in(0, T), \\
W(0) & =W_{0},
\end{aligned}
$$

where $H$ represents the right-hand side in (24) and $W_{0}$ is a given initial data. Next, we divide the time interval into subintervals $\left[t_{n}, t_{n+1}\right]$ with length $\Delta t=t_{n+1}-t_{n}$ for $n=0,1, \ldots$. We use the notation $W^{n}$ to denote the value of the solution $W$ at time $t_{n}$. The procedure to advance the solution from the time $t_{n}$ to the next time $t_{n+1}$ can be carried out as

$$
\begin{aligned}
W^{(1)} & =W^{n}+\Delta t H\left(W^{n}\right), \\
W^{(2)} & =\frac{3}{4} W^{n}+\frac{1}{4} W^{(1)}+\frac{1}{4} \Delta t H\left(W^{(1)}\right), \\
W^{n+1} & =\frac{1}{3} W^{n}+\frac{2}{3} W^{(2)}+\frac{2}{3} \Delta t H\left(W^{(2)}\right) .
\end{aligned}
$$

The scheme (26) is TVD, third-order accurate in time, and stable under the CFL condition 


$$
\Delta t \leq \min _{\Omega_{h}}\left(\frac{h_{e}}{(2 N+1) \max _{k=1, \ldots, 6}\left(\left|\lambda_{k}^{n}\right|\right)}\right),
$$

where $h_{e}$ is the diameter of the triangular element and $\lambda_{k}^{n}$ are the eigenvalues defined in (4), (6) and (7). The factor of $1 /(2 N+1)$ is an estimate of the CFL number required for stability (see [8]).

In order to prevent spurious oscillations at sharp fronts for the space discretization with $N \geq 1$, a slope limiter from [29] is applied at each step of the Runge-Kutta method described above. The details of this slope limiter can be found in [29] and they are not repeated here. Note that other slope limiters in [7] can also be applied without major conceptual modifications.

\section{NUMERICAL RESULTS}

In this section we present numerical results obtained for several test examples in the lock-exchange hydraulics. The main goals of this section are to illustrate the numerical performance of the DG method described above and to verify numerically its capability to solve the lock-exchange hydraulics on both flat and non-flat bottom beds. In all the computations reported herein, the gravitational constant $g=9.81 \mathrm{~m} / \mathrm{s}^{2}$, the Courant number $C$ is set to 0.7 and the time stepsize $\Delta t$ is adjusted at each step according to the stability condition (27). Furthermore, all the computations are made on a Pentium PC with two processors of $2 \mathrm{G}$ of RAM and $2.6 \mathrm{GHz}$. The codes only take the default optimization of the machine, i.e., they are not parallel codes.

\subsection{Lock-exchange problem on a flat bottom}

In this test example we consider a two-dimensional version of a one-dimensional problem of the lock-exchange hydraulics on a flat bottom in [4]. We solve the two-layer shallow water equations (1)-(2) in the rectangulardomain $[-3,3] \times[0,1]$ on a flat bottom (i.e., $B(x, y)=0$ ) and using the following initial conditions

$$
h_{1}(x, y, 0)=\left\{\begin{array}{l}
0, \text { if } x \leq 0, \\
1, \text { elsewhere }
\end{array} \quad h_{2}(x, y, 0)=\left\{\begin{array}{l}
1, \text { if } x \leq 0, \\
0, \text { elsewhere }
\end{array}\right.\right.
$$

and initially the flow is at rest i.e.,

$$
u_{1}(x, y, 0)=u_{2}(x, y, 0)=v_{1}(x, y, 0)=v_{2}(x, y, 0)=0 .
$$

The aim of this example is to examine the performance of the proposed DG method using different meshes. On the entry and exit boundaries, an exchange in the water discharges $q_{1, x}=-q_{2, x}$ and $q_{1, y}=-q_{2, y}$ is imposed. Here, the density ratio $\rho_{1} / \rho_{2}=0.95$, the order of polynomial approximation $N=3$ and four meshes are used in our simulations. The four meshes are depicted in Figure 1 and the statistics of these meshes are listed in Table 1 . Note that moving from the coarse mesh to the next fine mesh the number of elements and nodes are roughly doubled.

Table 1. Performance of the DG scheme for the lockexchange problem on a flat bottom using four meshes and $N=3$. The CPU times are given in seconds

\begin{tabular}{cccccc}
\hline & $\begin{array}{c}\text { \# of } \\
\text { elements }\end{array}$ & $\begin{array}{c}\text { \# of } \\
\text { nodes }\end{array}$ & Max $\boldsymbol{h}_{\mathbf{1}}$ & $\operatorname{Max} \boldsymbol{h}_{\mathbf{2}}$ & $\begin{array}{c}\text { CPU } \\
\text { time }\end{array}$ \\
\hline Mesh 1 & 1176 & 640 & 1.0230 & 1.0052 & 81 \\
Mesh 2 & 2366 & 1257 & 1.0228 & 1.0037 & 328 \\
Mesh 3 & 4691 & 2454 & 1.0165 & 1.0034 & 1276 \\
Mesh 4 & 9412 & 4851 & 1.0142 & 1.0031 & 4751 \\
\hline
\end{tabular}

The computed results for the water free-surface and the water interface are presented in Figure 2 at time $t=0.75$. It is clear that an increase in the mesh density results in an increase in the accuracy of the DG method for water heights. For example the numerical diffusion is more pronounced in the results on the coarsest mesh than the other results. Moreover, we remark that the computed results are in good agreement with alternative simulations using the one-dimensional model [4]. It is worth remarking that the first-order approximation of the eigenvalues in (6)-(7) gives complex values in some regions at certain simulation times. This explains the discontinuity appeared in the results obtained for the interface reported in [4]. The results obtained using the DG method do not show this drawback mainly because the maximum of the eigenvalues is used in the numerical fluxes (15). For visualizing the comparisons, we display in Figure 3 a cross section at the domain location $y=0.5$ of the results obtained for the proposed DG method using the four meshes. Under actual flow conditions, it is clear that the proposed DG method using the finest mesh Mesh 4 produces the best results compared to the other meshes. Results on the coarse meshes Mesh 1 and Mesh 2 are more dissipative than those obtained using the fine meshes Mesh 3 and Mesh 4. To further quantify the results for this test example we list in Table 1 the maximum values of the water free-surface, the water interface and the CPU times calculated in seconds. The clear indication from Table 1 is that the maximum value of the water heights slightly increases for the DG scheme on the coarse mesh Mesh 1, whereas the DG scheme on the fine mesh Mesh 4 requires more computational work than the other meshes. Apparently, the overall water flow features for this example are preserved with no spurious oscillations appearing in the results obtained using the proposed DG method. Obviously, the computed results verify the stability and the shock capturing properties of the proposed DG method. 

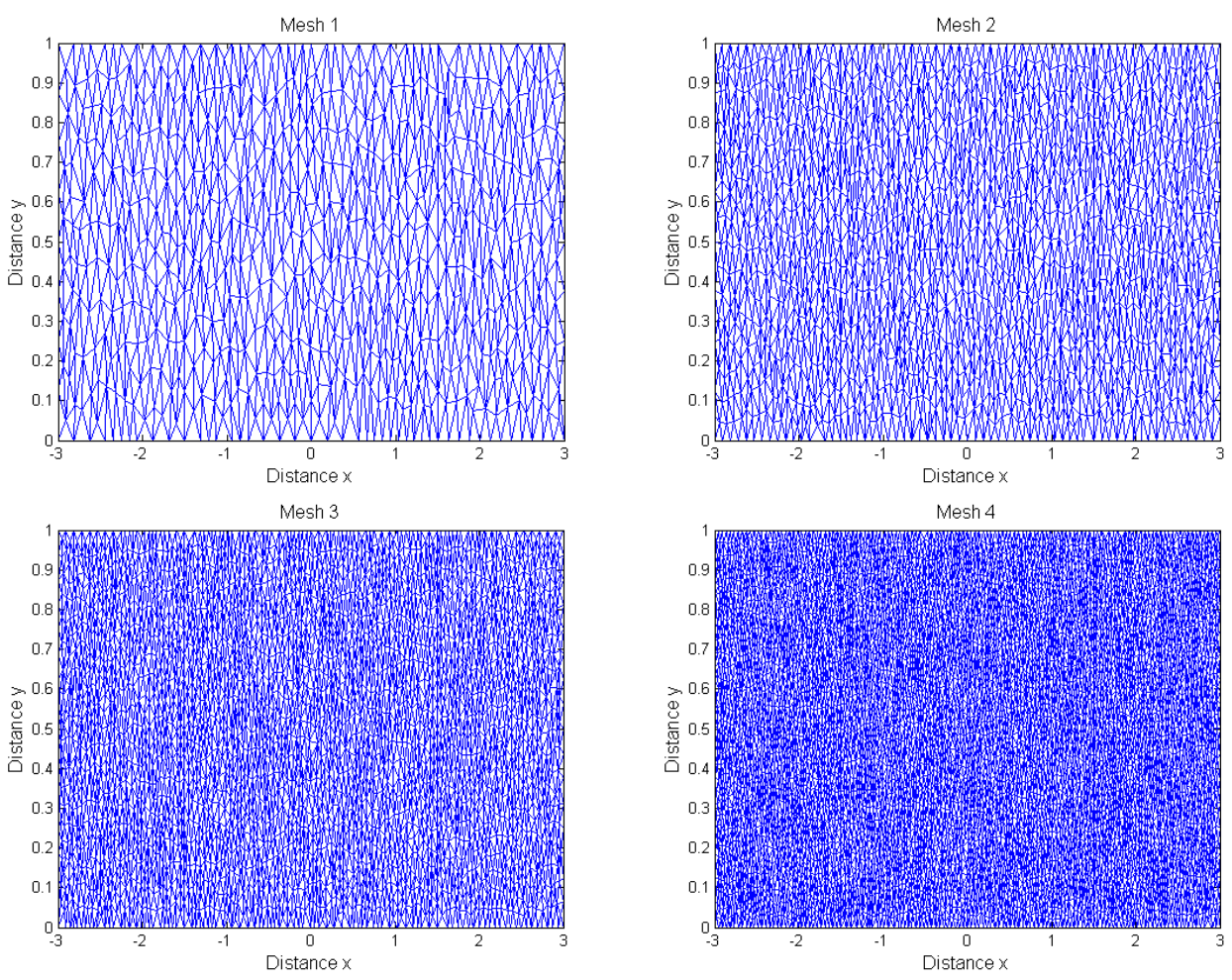

Fig 1: The meshes used for the lock-exchange problem on a flat bottom.
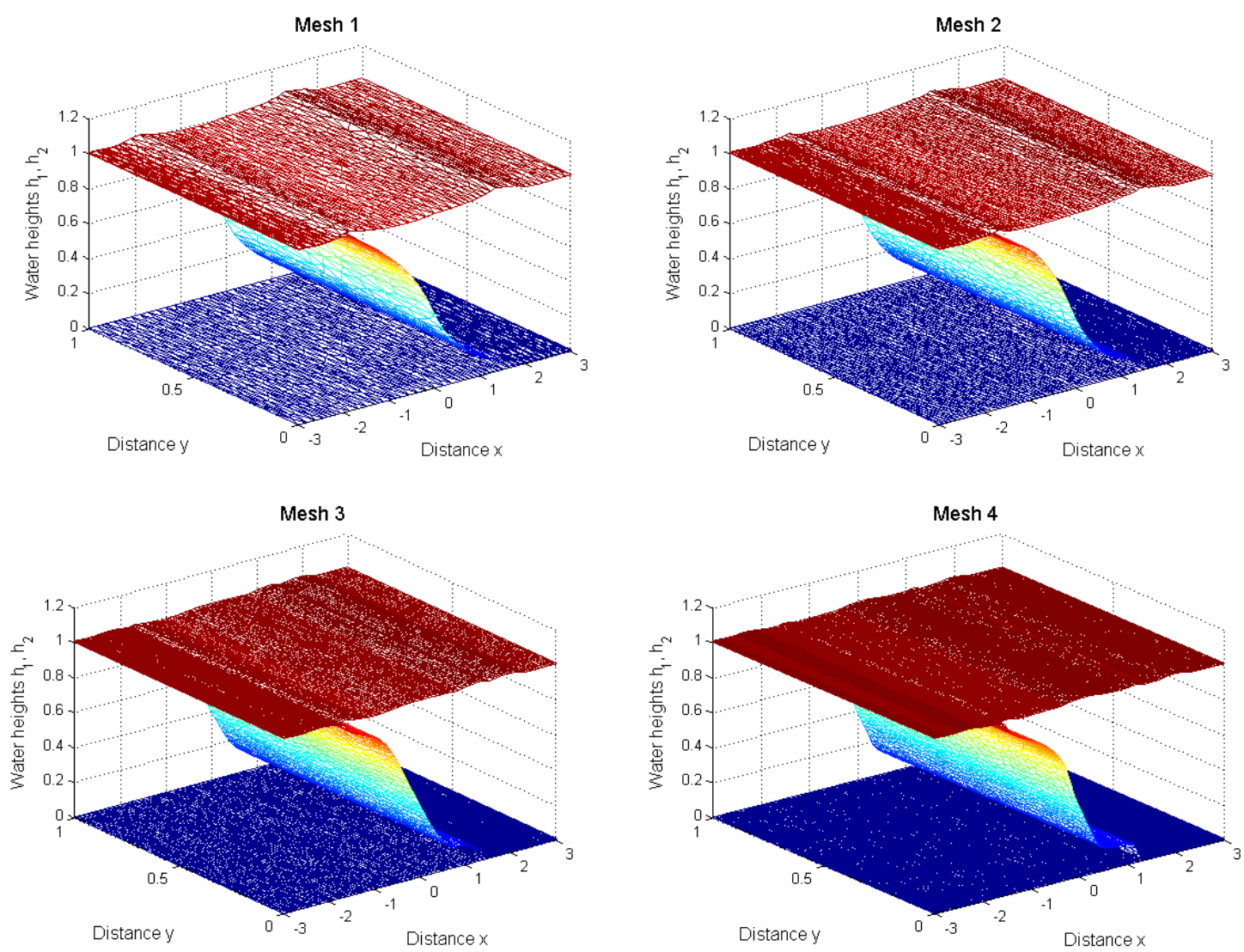

Fig 2: Water heights for the lock-exchange problem on a flat bottom at time $t=0.75$ using four different meshes. 


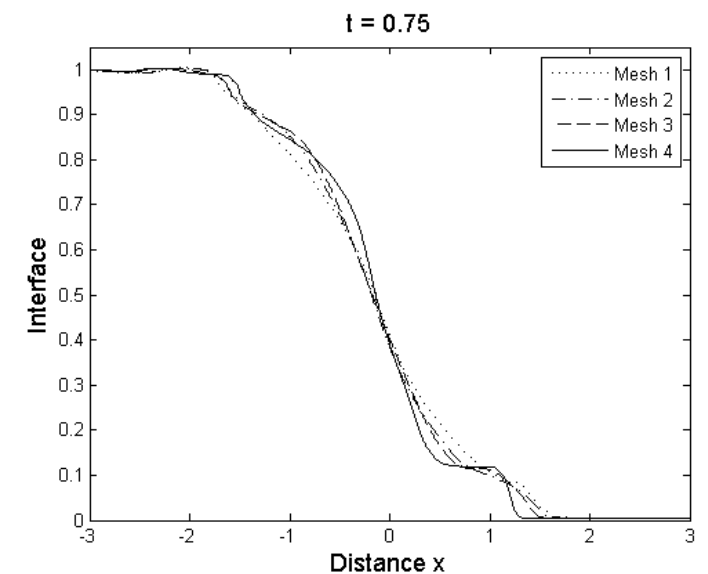

Fig 3: Cross section of the water interface for the lockexchange problem on a flat bottom at the location $y=0.5$ and time $\mathbf{t}=\mathbf{0 . 7 5}$.

\subsection{Lock-exchange problem on a non-flat bottom}

We consider a test example of lock-exchange hydraulics over a hump. This problem is inspired by the works of [12, 26, 5] where tidal and exchange flow in the Strait of Gibraltar was studied using the two-layer shallow water equations. Here, the two-layer shallow water equations (1)-(2) are solved over a bottom topography considered to be a Gaussian-shaped function defined as

$$
B(x, y)=\exp \left(-x^{2}\right) .
$$

The two layers are initially separated and the lighter water is on the left while the heavier one is on the right i.e.,

$$
\begin{aligned}
& h_{1}(x, y, 0)= \begin{cases}2-B(x, y), & \text { if } x \leq 0, \\
0, & \text { elsewhere, }\end{cases} \\
& h_{2}(x, y, 0)= \begin{cases}0, & \text { if } x \leq 0, \\
2-B(x, y), & \text { elsewhere, }\end{cases}
\end{aligned}
$$

and

$$
u_{1}(x, y, 0)=v_{1}(x, y, 0)=u_{2}(x, y, 0)=v_{2}(x, y, 0)=0,
$$

The computational domain is $[-3,3] \times[0,1]$ and the boundary conditions at the downstream and the upstream of the channel are imposed on the water discharges $q_{1}=$ $\left(q_{1, x}, q_{1, y}\right)^{T}$ and $q_{2}=\left(q_{2, x}, q_{2, y}\right)^{T}$ as

$$
\int_{\Gamma}\left(q_{1}(\gamma)+q_{2}(\gamma)\right) \cdot \mathbf{n}(\gamma) d \gamma=0,
$$

where $\Gamma$ refers to the open boundaries of the channel located at $x=-3$ and $x=3$, compare [26] for more details on the description of this test example.
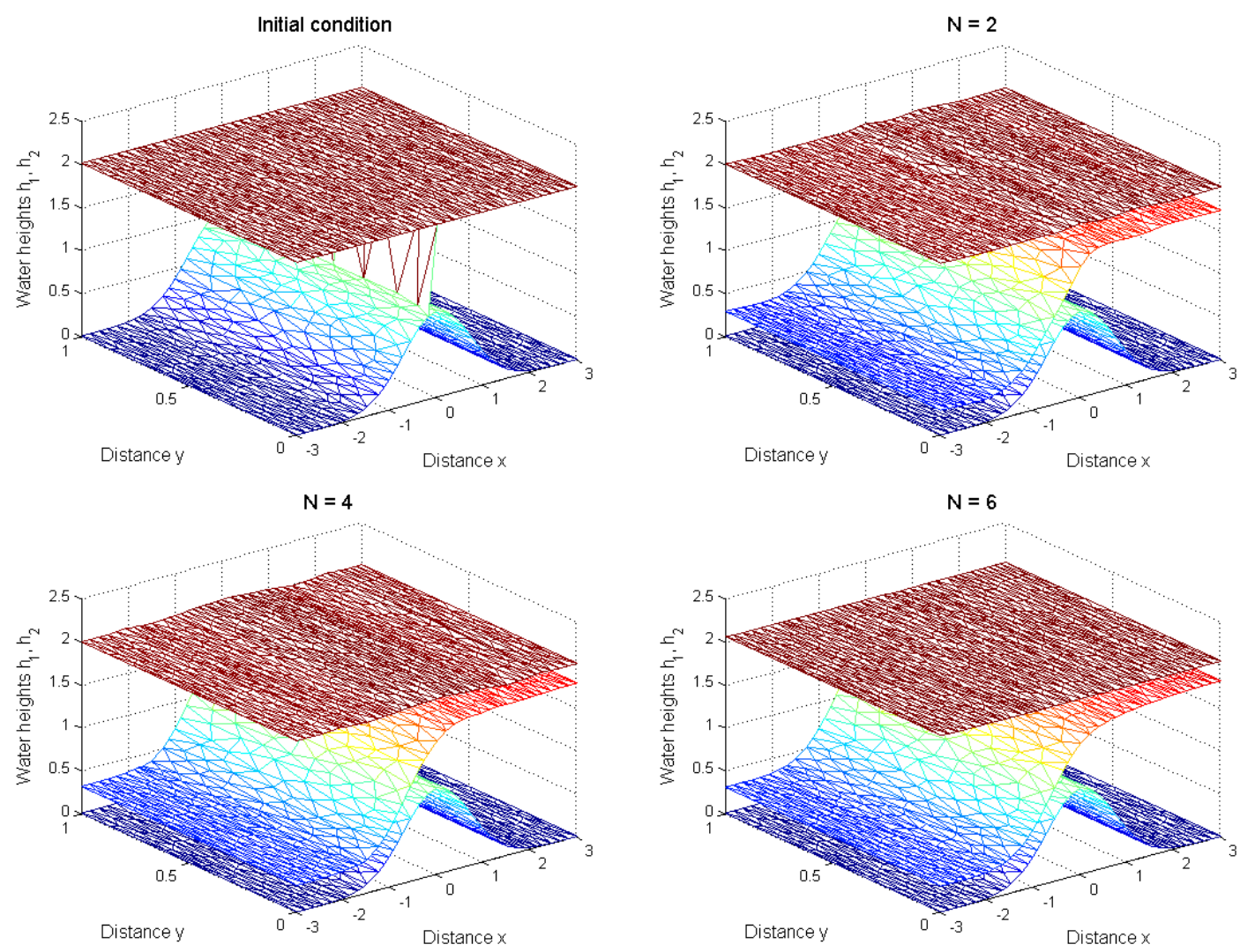

Fig 4: Water heights for the lock-exchange problem on a non-flat bottom using different orders of polynomial approximation. 


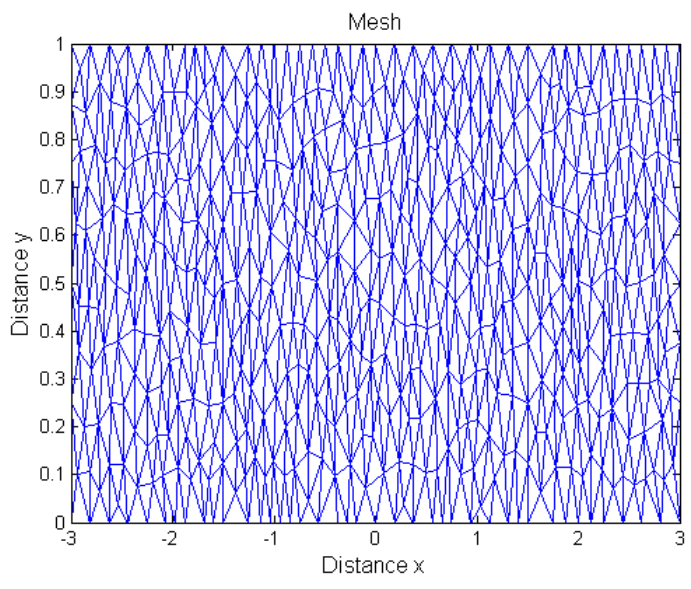

Fig 5: The mesh used for the lock-exchange problem on a non-flat bottom.

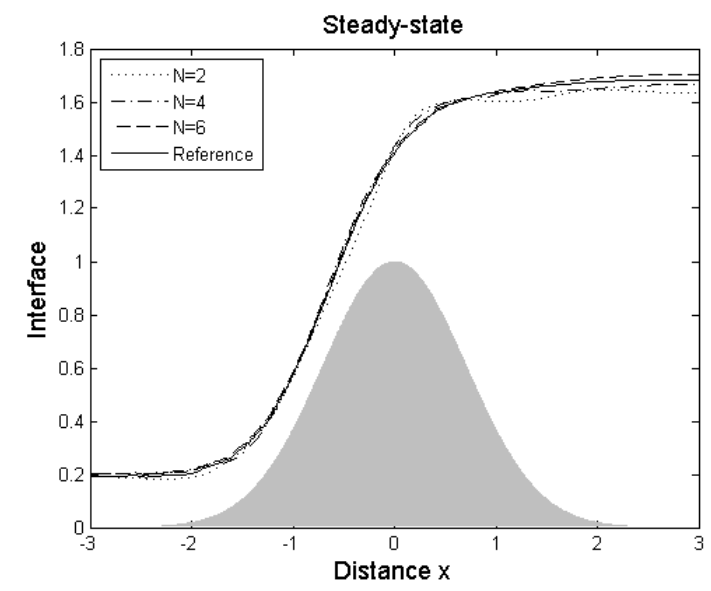

Fig 6: Cross section of the water interface for the lock-exchange problem on a non-flat bottom at the location $y=$ 0.5

The density ratio is $\rho_{1} / \rho_{2}=0.98$ and it is expected that the heavier water propagates to the upstream, while the lighter one moves to the downstream. The solution is also expected to converge to a smooth steady state. The purpose of this example is to verify the response of the DG method for the variation of the order of polynomial approximation $N$. To this end the computational domain is discretized into 914 elements and the polynomial degree $N$ is set to 2, 4 and 6. Only steadystate solutions are presented for this test example. In Figure 4 we present the steady-state numerical results for the water free-surface and the water interface using the selected orders of polynomial approximation. In this figure, we have also included the initial conditions along with the bottom bed. The water free-surface creates a strong interaction with the hump, resulting in the formation of strong and weak shocks. By using different values of $N$, high resolution is clearly obtained in those regions where the gradients of the water depth are steep such as the moving fronts. Apparently, the overall flow pattern for this example is preserved with no spurious oscillations appearing in the results by DG method using fixed meshes. Obviously, the obtained results verify the stability and the shock capturing properties of the proposed DG method. In addition the proposed DG method performs well for this test problem since it does not diffuse the moving fronts and no spurious oscillations have been observed when the water flows over the hump.

Table 2. Performance of the DG scheme for the lockexchange problem on a non-flat bottom using different polynomial degrees and a mesh of 914 elements. The CPU times are given in seconds.

\begin{tabular}{cccc}
\hline & Max $\boldsymbol{h}_{\mathbf{1}}$ & Max $\boldsymbol{h}_{\mathbf{2}}$ & CPU time \\
\hline $\boldsymbol{N}=\mathbf{2}$ & 1.7060 & 1.727 & 1223 \\
$\boldsymbol{N}=\mathbf{4}$ & 1.7296 & 1.775 & 4228 \\
$\boldsymbol{N}=\mathbf{6}$ & 1.7483 & 1.802 & 10729 \\
\hline
\end{tabular}

For comparison reasons, cross sections of the water interface at the channel location $y=0.5$ are shown in Figure 6 in which we have included a reference solution [22]. These results are very similar to the ones obtained in $[12,5]$. It should be stressed that no interface instabilities have been observed in this example, even though initially $h_{1}=0$ for $x>0$ and $h_{2}=0$ for $x<0$ and at small times, either $h_{1}$ or $h_{2}$ is (almost) zero in a significant part of the computational domain. One of the key stability factors here is the ability of our DG method to preserve the positivity of each layer depth. For further comparisons we summarize in Table 2 the maximum values of the water heights $h_{1}$ and $h_{2}$ along with the corresponding CPU times for the three different orders $N=2,4$ and 6 . It is clear that the considered orders of polynomial approximation give roughly the same maximum values for both water heights. However, in terms of efficiency, the proposed DG method using $N=6$ requires two to three times more computational work than the DG method using $N=4$.

\section{CONCLUSIONS}

In this paper we have presented a discontinuous Galerkin method for the numerical simulation of lock-exchange hydraulics. The model consists of solving the two-layer shallow water equations on both flat and non-flat topography. Specific details were given on the implementation of the discontinuous Galerkin method using unstructured meshes. The method combines some properties of the finite element and the finite volume techniques, resulting on a very attractive method because of its formal high-order accuracy and its ability to capture discontinuities without producing spurious oscillations. Numerical results and applications have been illustrated for two test problems of lock-exchange hydraulics. The presented results demonstrate the accuracy of the method and its capability to simulate lock-exchange hydraulics in the hydrodynamic regimes considered. We conclude with some comments on the current development of this discontinuous Galerkin method, in terms of both physical and numerical features that will be implemented. In this paper, we have only considered source terms due to the bottom topography. However, in many lock-exchange hydraulic scenarios friction losses and viscous terms, which interact with the hydraulics. through the introduction of stress terms in the momentum equations, can be the dominant force in the two-layer shallow water flows. Therefore, future work will involve inclusion of 
viscous coupling a wave model component into the modelling system to include the effects of bottom friction, wind stress, eddy viscosity, and Coriolis force in the hydraulics.

\section{ACKNOWLEDGMENTS}

Financial support provided by MULIT and MHYCOF projects is gratefully acknowledged.

\section{REFERENCES}

[1] R. Abgrall, S. Karni, Two-layer shallow water systems: a relaxation approach, SIAM J. Sci. Comput. 31 (2009) $1603-1627$.

[2] V. Aizinger, C. Dawson, A discontinuous Galerkin method for two-dimensional flow and transport in shallow water, Advances in Water Resources 25 (2002) 67-84.

[3] Y. Bazilevs, T. Hughes, Weak imposition of Dirichlet boundary conditions in fluid mechanics, Computers \& Fluids, in press 36 (2007) 12-26.

[4] F. Bouchut, T. Morales, An entropy satisfying scheme for two-layer shallow water equations with uncoupled treatment, M2AN Math. Model. Numer. Anal. 42 (2008) 683-698.

[5] M. Castro, J. García -Rodriguez, J. González -Vida, J. Macias, C. Parés, M. Vázquez-Cendón, Numerical simulation of two-layer shallow water flows through channels with irregular geometry, J. Comp. Physics. 195 (2004) 202-235.

[6] B. Cockburn, G.E. Karniadakis, C.W.S. (eds.), Discontinuous Galerkin methods. Theory, computation and applications, Lecture Notes in Computational Science and Engineering, 11. Springer-Verlag, Berlin, 2000.

[7] B. Cockburn, C. Shu, The Runge-Kutta discontinuous Galerkin method for conservation laws V: Multidimensional systems, J. Comput. Phys. 141 (1998) 199224.

[8] B. Cockburn, C.W. Shu, The Runge-Kutta discontinuous Galerkin methods for convection-dominated problems, Journal of Scientific Computing. 16 (2001) 173-261.

[9] M. Dubiner, Spectral methods on triangles and other domains, Journal of Scientific Computing 6 (1991) 345390.

[10] M. Dudzinski, M. Medvidova, Well-balanced pathconsistent finite volume EG schemes for the two-layer shallow water equations, Computational Science and High Performance Computing. IV (2009) 121-136.

[11] C. Eskilsson, S.J. Sherwin, A triangular spectral/hp discontinuous Galerkin method for modelling $2 \mathrm{~d}$ shallow water equations, Int. J. Numer. Methods Fluids. 45 (2004) 605-623.

[12] D. Farmer, L. Armi, Maximal two-layer exchange over a sill and through a combination of a sill and contraction with barotropic flow, J. Fluid Mech. 164 (1986) 53-76.

[13] T.W. F.X. Giraldo, A nodal triangle-based spectral element method for the shallow water equations on the sphere, Journal of Computational Physics 207 (2005) $129-150$.
[14] F.X. Giraldo, T. Warburton, A high-order triangular discontinuous Galerkin oceanic shallow water model, Int. J. Numer. Meth. Fluids 56 (2008) 899-925.

[15] J. Hesthaven, From electrostatics to almost optimal nodal sets for polynomial interpolation in a simplex, SIAM J. Numer. Anal. 35 (1998) 655-676.

[16] J. Hesthaven, T.Warburton, High-order nodal methods on unstructured grids. I. Time-domain solution of maxwells equations, J Comp Phys 181(1) (2002) 186221.

[17] T. Hughes, G. Scovazzi, P. Bochev, A. Buffa, A multiscale discontinuous Galerkin method with the computational structure of a continuous Galerkin method, Computer Methods in Applied Mechanics and Engineering 195 (2006) 2761-2787.

[18] C.W.S. J. Qiu, B. C. Khoo, A numerical study for the performance of the Runge-Kutta discontinuous Galerkin method based on different numerical fluxes, J. Comput. Phys. 212 (2006) 540-565.

[19] S.G. J.S. Hesthaven, D. Gottlieb, Spectral Methods for Time-Dependent Problems, Cambridge University Press, Cambridge, 2006.

[20] T. Koornwinder, Two-variable analogues of the classical orthogonal polynomials, in: R.A. Askey (Ed.), Theory and Applications of Special Functions, Academic Press, San Diego, 1975.

[21] E. Kubatko, J. Westerink, C. Dawson, A. Buffa, hp discontinuous Galerkin methods for advection dominated problems in shallow water flow, Computer Methods in Applied Mechanics and Engineering 196 (2006) $437-$ 451.

[22] A. Kurganov, G. Petrova, Central-upwind schemes for two-layer shallow water equations, SIAM J. Sci. Comput. 31 (2009) 1742-1773.

[23] W. Lee, A. Borthwick, P. Taylor, A fast adaptive quadtree scheme for a two-layer shallow water model, J. Comp. Physics. 230 (2011) 4848-4870.

[24] R. Leveque, Finite Volume Methods for Hyperbolic Problems, Cambridge University Press, Cambridge, 2002.

[25] R.V. M.A. Taylor, B.A. Wingate, An algorithm for computing fekete points in the triangle, SIAM J. Numer. Anal. 38 (2000) 1707-1720.

[26] J. Macías, C. Parés, M. Castro, Improvement and generalization of a finite element shallow water solver to multi-layer systems, Int. J. Num. Methods Fluids. 31 (1999) 1037-1059.

[27] J. Proriol, Sur une famille de polynomes à deux variables orthogonaux dans un triangle, C.R. Acadamic Science, Paris 257, 1957.

[28] C. Shu, Total variation diminishing time discretizations, SIAM J. Sci. Stat. Comput. 9 (1988) 1073-1084.

[29] S. Tu, S. Allibadi, A slope limiting procedure in discontinuous Galerkin finite element method for gas dynamics applications, International Journal of Numerical Analysis and Modeling. 2 (2005) 163-178.

[30] C. Vreugdenhil, Two-layer shallow-water flow in two dimensions, a numerical study, J. Comp. Physics. 33 (1979) 169-184. 